\title{
Novel Grid Dynamic Economic Dispatch Method Considering Response Characteristic of Intelligent Power Consumption Community
}

\author{
Wang Shiqian ${ }^{1, *}$, Zhang $\mathrm{Yu}^{2}$, Song Xiaokai ${ }^{1}$, Guo Jianyu ${ }^{1}$ and Liu Yang $^{1}$ \\ ${ }^{1}$ Henan Electric Power Company Economic Research Institute, Zhengzhou 450052, Henan in China \\ ${ }^{2}$ State Key Laboratory of Alternate Electrical Power System with Renewable Energy Sources of North China Electric Power University, \\ Beijing 102206, China
}

\begin{abstract}
Nowadays smart grid and intelligent power consumption community develop rapidly, and the interactive coupling of power system with intelligent community has become more in-depth. Based on differential evolution algorithm, a grid dynamic economic dispatch method with the intelligent community is proposed in this realistic context. The objective function is to minimize generation cost. And multiple constraint conditions are power balance, reserve capacity of electric power system, generator output, generator climbing speeds and et al. The accuracy and validity of the method are validated through analyzing the example of a power system including two intelligent communities.
\end{abstract}

\section{Introduction}

Intelligent power consumption community is an important part for smart grid. Residential customers can achieve the synchronous management of home energy and information by configuring smart sockets, smart infrared remote control devices and interactive software. Widespread adoption of intelligent power consumption community not only applies higher power reliability and satisfies user's multiple requirement, but also maximizes energy, economic and environment benefits ${ }^{[1-4]}$. For grid, intelligent power consumption community effect mainly includes economic load distribution during normal operation, regulating peak during peak load, grid support in emergency situations. Intelligent power consumption community participates in grid dispatch in the form of micro grid and co-operates with grid, which is one of the development directions of smart grid. And the smart grid develops rapidly, intelligent power consumption community forms fast and the interactive coupling effects between intelligent power consumption community and power system have become more indepth. So in this realistic context, how to combine the impact of intelligent power consumption community access on grid and establish a reasonable dynamic economic dispatch model has become a pressing problem that needs to be solved urgently.

\section{Grid dynamic economic dispatch method including intelligent power consumption community}

\footnotetext{
* Corresponding author:* Wang shiqian: wsq0204@163.com
}

\subsection{Formatting the title, authors and affiliations}

The basic idea of dynamic economic dispatch is to consider demand response characteristic of intelligent power consumption community from the perspective of grid operators with the goal that minimizes generation cost and multiple constraints that include power balance, reserve capacity of electric power system, generators output, unit ramp rate and et al. And a grid dynamic economic dispatch is established to obtain reasonable output for each generator set at different times.

Based on above analysis, the following objective functions can be established for the grid including intelligent power consumption community:

$$
\min C=F_{G}+F_{M G}
$$

In the formula, $C$ is the total generation cost, $F_{G}$ is the central-dispatch units operation cost, $F_{M G}$ is the cost of participation in scheduling for intelligent community.

The formula corresponding to the objective function is:

$$
\begin{gathered}
F_{G}=\sum_{t=1}^{T} \sum_{i=1}^{N_{G}}\left(\alpha_{i} P_{G i, t}^{2}+\beta_{i} P_{G i, t}+\gamma_{i}\right) \\
F_{M G}=\sum_{t=1}^{T} \sum_{j=1}^{N_{M G}}\left(\lambda_{j, t} P_{M G j, t}\right)
\end{gathered}
$$

In the formula, $T$ is scheduling period, $N_{G}$ is the number of central-dispatch units, $\alpha_{i}, \beta_{i}, \gamma_{i}$ are quadratic function coefficient for $i$ th generation cost, $P_{G i, t}$ is active power output of $i$ th generator in interval " $\mathrm{t}$ ". $N_{M G}$ is the number of intelligent power consumption communities; 
when $P_{M G j, t}>0, P_{M G j, t}$ is active power output for intelligent power consumption community to grid and $\lambda_{j, t}$ is the price for intelligent power consumption community to grid; when $P_{M G j, t}<0, P_{M G j, t}$ is active power output for grid to intelligent power consumption community and $\lambda_{j, t}$ is the price for grid to intelligent power consumption community.

Constrains corresponding to objective functions are:

A) power balance:

$$
P_{D, t}+R_{D, t}=\sum_{i=1}^{N_{G}} P_{G i, t}
$$

B) reserve capacity of electric power system:

$$
P_{G i, t-1}-P_{G i, t} \leq \Delta D P_{G i}
$$

C) output limits of conventional generators:

$$
P_{G i \min } \leq P_{G i, t} \leq P_{G i \max }
$$

D) dynamic constraint of climbing speeds for centraldispatch units:

$$
\left\{\begin{array}{l}
P_{G i, t}-P_{G i, t-1} \leq \Delta U P_{G i} \\
P_{G i, t}-P_{G i, t-1} \leq \Delta D P_{G i}
\end{array}\right.
$$

E) dynamic constraint for intelligent power consumption community output:

$$
P_{M G j \min , t} \leq P_{M G j, t} \leq P_{M G j \max , t}
$$

In the formula, $P_{D, t}, R_{D, t}$ are load demand and standby load demand in interval " $\mathrm{t}$ ", , respectively; $P_{G i \max }, P_{G i \min }$ are maximum and minimum output of ith generator, respectively; $\Delta U P_{G i}, \Delta D P_{G i}$ are generator ramp up and ramp down limits in unit time, respectively; $P_{M G j \text { max }, t}, P_{M G j \min , t}$ are maximum and minimum output limits for $\mathrm{jth}$ intelligent power consumption community in interval " $t$ ", respectively.

\subsection{Solving the grid dynamic economic dispatch model with intelligent power consumption community based on differential evolution algorithm}

Differential evolution algorithm (DE) is a novel intelligent optimization approach based on populationbased optimization, which has many advantages such as high effectiveness, convergence, robustness and et al ${ }^{[5-6]}$. So this paper uses grid dynamic economic dispatch model with intelligent power consumption community based on differential evolution algorithm.

)1( Input of original parameters. These parameters include population size $n$, solution-space dimension $n p$, mutation factor $F$ and crossover factor $C R$, also include maximum and minimum output constraint values, upgoing and downgoing ramping constraint values, upper and lower predicting limits of each intelligent power consumption community output, sale and purchase price of each intelligent power consumption community.
(2) Initial population. Initial pollution is generated by output limits of each generator and predicting limits of each intelligent power consumption community output:

$$
X_{i, j}=X_{i, j \min }+\operatorname{rand}\left(X_{i, j \max }-X_{i, j \min }\right)
$$

In the formula, rand() represents a uniformly distributed random variable within the range $[0,1]$.

(3) The calculation of objective function. The minimum generation cost is calculated by bring each individual in the population into the objective function.

(4) Constraints processing. Each individual in the population is tested by bringing it into constraint condition. If it cannot satisfy constraint condition, objective function with additional penalty item will be eliminated in the selection operation and the individual which satisfies constrain condition stays.

(5) Selection operation. Each individual minimum generation cost is compared to that of its corresponding objective function value in the last population. And the individual that has less generation cost will stay.

(6) Evolutionary operation. After selection operation, whether the iteration number satisfies stopping criterion for iteration is judged. If it satisfies, optimum individual and objective function value are output. Otherwise, evolutionary operation including variation, cross and so on is done to produce the next individual based on formula (10) and (11). And then return to Step 3 again.

$$
\mathrm{V}_{i, j}^{\mathrm{G}+1}= \begin{cases}\mathrm{U}_{\mathrm{i}}^{\mathrm{G}+1}=\mathrm{X}_{r 1}^{\mathrm{G}}+\mathrm{F} \bullet\left|\mathrm{X}_{r 2}^{\mathrm{G}}-\mathrm{X}_{r 3}^{\mathrm{G}}\right| & \operatorname{rand}(j)<C R \text { or } j \neq \operatorname{randr}_{i} \\ \mathrm{X}_{i, j}^{\mathrm{G}} & \operatorname{rand}(j) \geq C R \text { or } j=\operatorname{randr}_{i}\end{cases}
$$

In the formula, $\mathrm{X}_{r 1}^{\mathrm{G}} 、 \mathrm{X}_{r 2}^{\mathrm{G}} 、 \mathrm{X}_{r 3}^{\mathrm{G}}$ are three individuals randomly selected from the $G$ generation population except $\mathrm{X}_{i}^{\mathrm{G}}$ and $r 1 \neq r 2 \neq r 3, F$ is a mutation factor, $\mathrm{U}_{\mathrm{i}}^{\mathrm{G}+1}$ is a mutation individual corresponding to $\mathrm{X}_{i}^{\mathrm{G}}, \operatorname{rand}(j)$ is a random number selected in $[0,1]$; $C R \in[0,1]$ is a crossover factor; $r a n d r_{i}$ is a integer randomly selected in $[0, \mathrm{np}]$.

\section{Example and Analysis}

In order to verify the feasibility and superiority of coordination model between intelligent district and grid, this paper uses a power system with six generating units as an example to calculate, of which two units are intelligent power consumption communities. $\alpha_{i}, \beta_{i}, \gamma_{i}$ of four conventional power units are $0.00712,22.26,370$; $0.00413,25.92,660 ; 0.00222,27.27,665 ; 0.00173$, 27.79, 670, respectively; maximum output, minimum output, upgoing ramping rate, downgoing ramping rate are $80,20,20,20 ; 55,10,20,20 ; 55,10,20,20 ; 55,10,20,20$; intelligent power consumption communitiess report the 24 hours upper and lower predicting limits according to the capacity of distributed power in the districts and their own load predicting data as shown in Figure 2. This example system load curve is shown as Figure 3. And load reserve of each period is $10 \%$ of the system load during this period. 


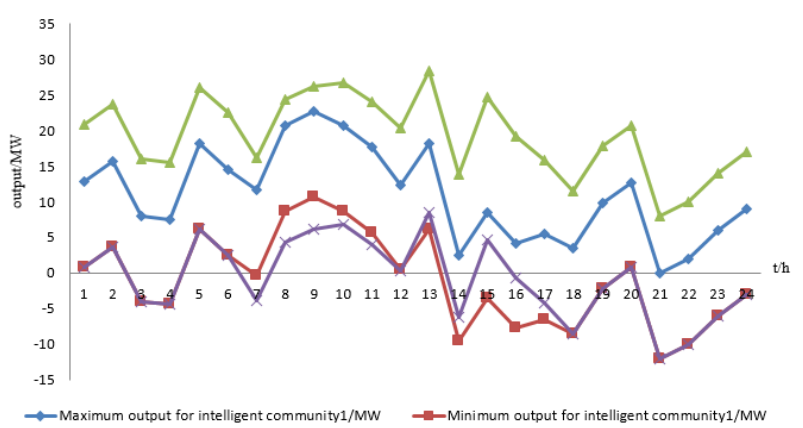

-Maximum output for intelligent community $2 / \mathrm{MW}$-Minimum output for intelligent community2/MW

Fig.1. Intelligent communities report the 24 hours upper and lower predicting limits

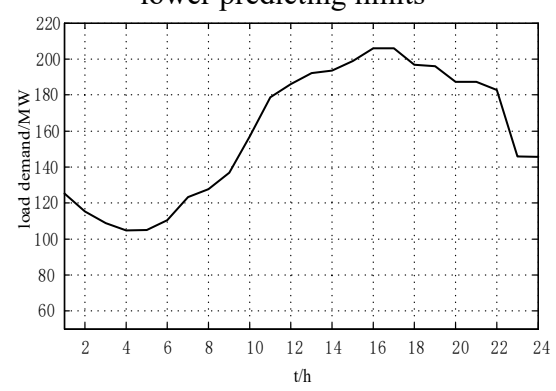

Fig. 2. Example system load curve

The dimension of solution space is set as 120 , population size is 600 , evolution to $800^{\text {th }}$ generation converge, mutation factor $F$ is 0.5 , crossover factor $C R$ is 0.9 ; sale prices from intelligent power consumption communities 1 and 2 to grid is $\$ 30 / \mathrm{MW}$ and $\$ 28 / \mathrm{MW}$, respectively; and the prices purchased from grid operators both are $\$ 24 / \mathrm{MW}$.

The evolution curves of generator output and total generation cost by grid operators using dynamic economic dispatch model in Section 2 are shown as Figure 3., respectively. The final total generation cost is $\$ 152,380$ as shown in Figure 4.

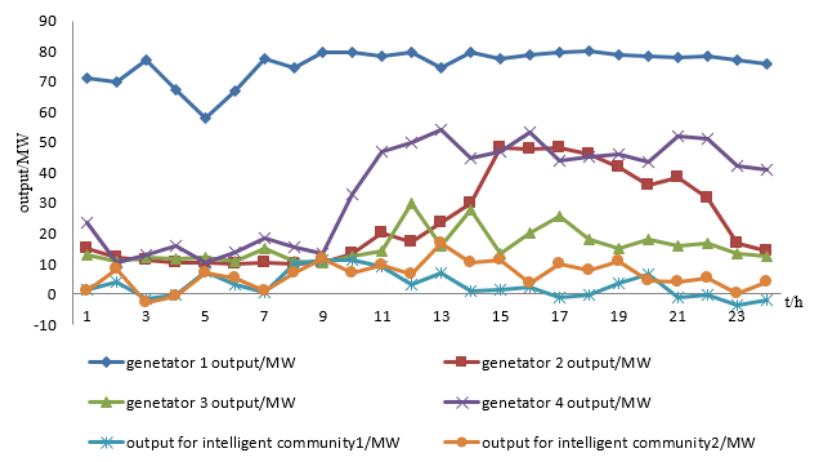

Fig. 3. Generator output

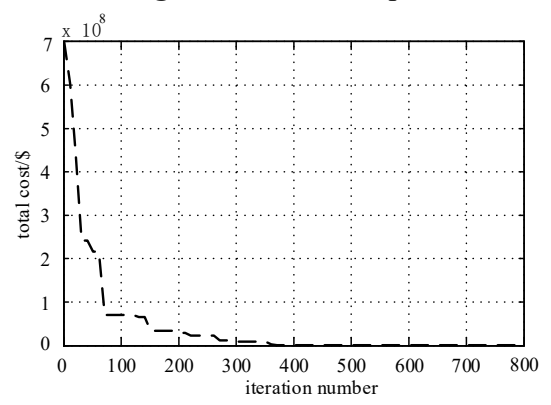

Fig. 4. Evolution curve of generation cost
The curves of intelligent power consumption communities output are shown as Figure 5. and Figure 6, respectively. Positive value indicates that intelligent power consumption communities sell power to grid operators, which is characteristic of power source. Negative value indicates that intelligent power consumption communities purchase power to grid operators, which is load characteristic.

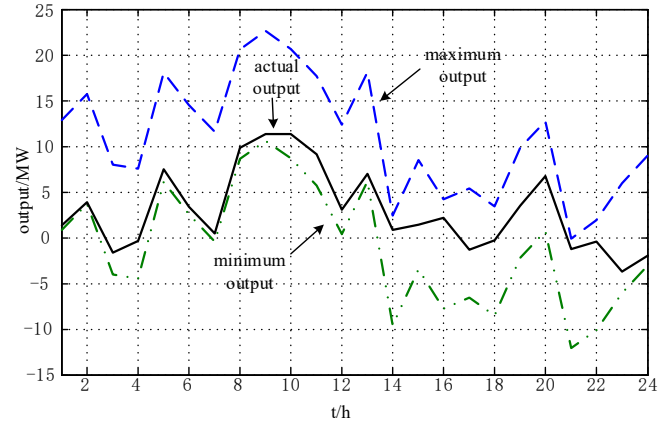

Fig. 5. Output curve of intelligent community 1

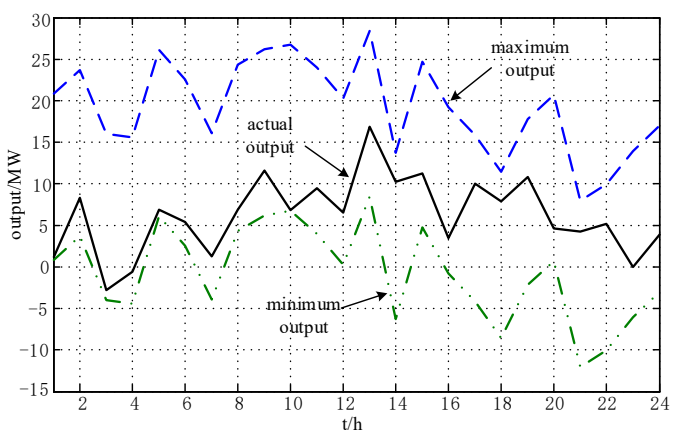

Fig. 6. Output curve of intelligent community 2

From Figure 5. and Figure 6, it can be seen that under normal operating condition intelligent power consumption community can according to real-time load sell power to grid operators during peak load and purchase power from grid operators, which indicates that intelligent power consumption community has characteristic of power source and load, flexible operation modes, and significant economic benefits. For grid operators, the participation of intelligent power consumption community in dispatch has played a role in peak load shifting and helping the grid to operate safely and economically.

\section{Conclusion}

This paper establishes a grid dynamic economic dispatch considering response characteristic of intelligent community, which achieves mutual benefit between intelligent community and grid operators. It has important practical significance for a secure economic grid with a large number of intelligent power consumption communities.

\section{References}

1. Lu Jun, Zhu Yan-ping, Peng Wen-hao, et al. Interactive Demand Response Method of Smart Community Considering Clustering of Electricity 
Consumption Behavior, Automation of Electric Power System 2017,41(17):113-120

2. Jorge H., Antunes C.H., Martins A.G. A multiple objective decision support model for the selection of remote load control strategies . 2000,2(2):865-872.

3. Li Tongzhi. Technical Implications and Development Trends of Flexible and Interactive Utilization of Intelligent Power. Automation of Electric Power System, 2012,36(2):11-17

4. Sheng Wanxing, Sun Changkai, SUN Jun-ping, et al. Characteristics and Research Framework of Automated Demand Response in Smart Utilization. Automation of Electric Power System, 2013, 37(23): 1-7.

5. Chen Guangyu, Ding Xiaoqun, Bian Erman. Dynamic Differential Evolution Algorithm Based on Chaotic Sequences for Dynamic Economic Dispatch Problem of Power System. Electric Power, 2016,49(6):101-106

6. Kang Zhongjian, Zi Shuwei. Research on the Reactive Power Optimization of Oil-Field Regional Distribution Network Based on Differential Evolution. Transactions of China Electrotechnical Society, 2013,33(6):226-231 